\title{
Leaderless Formation Control for Multiple Autonomous Vehicles
}

\author{
Wei Ren* \\ Department of Electrical and Computer Engineering, Utah State University, Logan, UT, 84322, USA \\ YangQuan Chen ${ }^{\dagger}$ \\ Department of Electrical and Computer Engineering, Utah State University, Logan, UT, 84322, USA
}

This paper develops a leaderless formation control strategy based on emergent consensus algorithms. The proposed strategy only requires local neighbor-to-neighbor information exchange between vehicles and does not assume the existence of any explicit leaders in the team. The proposed strategy is then applied to a micro air vehicle (MAV) formation flying scenario as a proof of concept.

\section{Nomenclature}

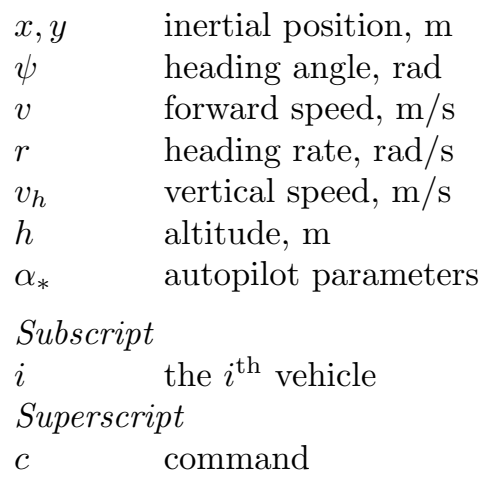

\section{Introduction}

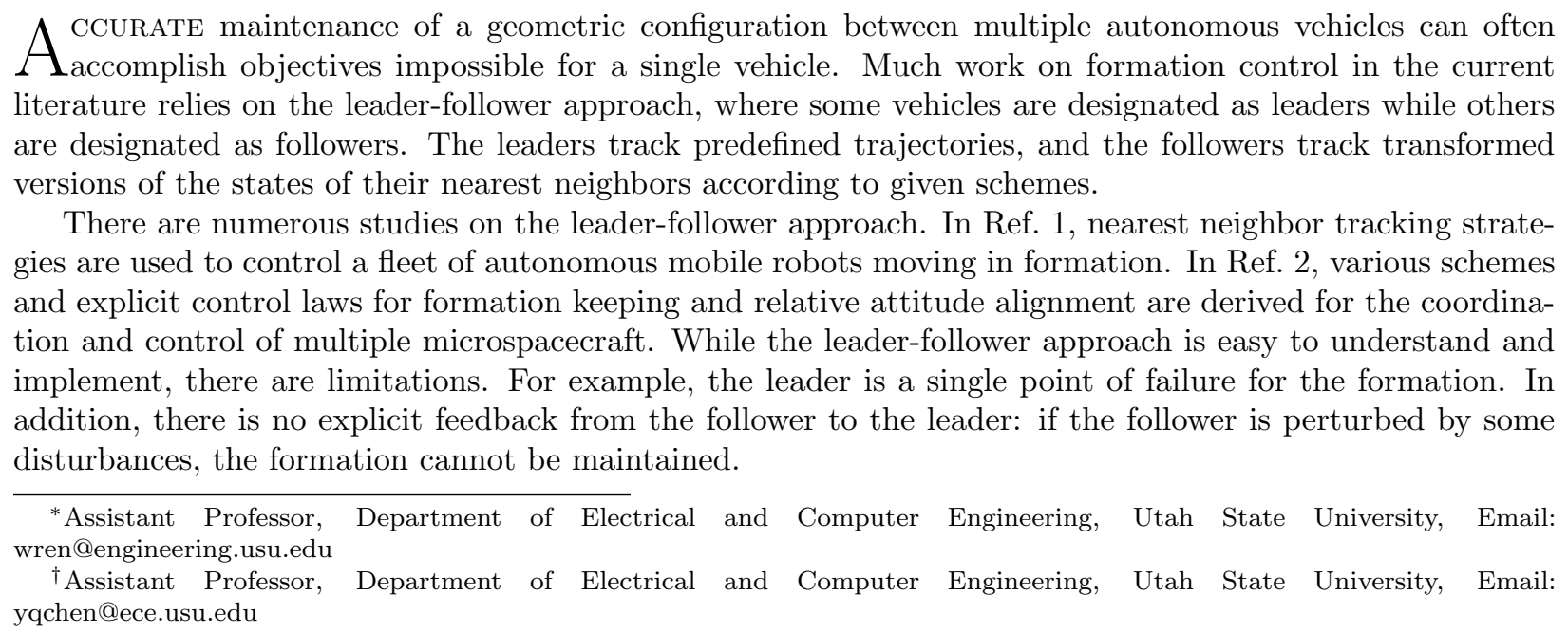


Related to the leader-follower approach are the consensus type problems in cooperative control of mobile autonomous agents, where each agent in a team updates its information state based on the information states of its local neighbors in such a way that the final information state of each agent converges to a common value. The research on consensus algorithms has been reported in Refs. $3-5$, to name a few. Those algorithms take the form of single integrator dynamics. Extensions to second-order dynamics under undirected information flow and directed information flow are discussed in Refs. 6,7 and Ref. 8 respectively.

In this paper, we propose a leadless formation control strategy based on consensus algorithms. In particular, no explicit leaders exist in the team and the proposed strategy only requires local neighbor-to-neighbor information exchange between vehicles. As a result, the proposed strategy overcomes a single point of failure for the formation and allows explicit feedback between neighboring vehicles through information exchange. The proposed strategy is also applied to a micro air vehicle (MAV) formation flying scenario as a proof of concept.

\section{Background and Preliminaries}

\section{A. Graph Theory}

It is natural to model information exchange between vehicles by directed/undirected graphs. A digraph (directed graph) consists of a pair $(\mathcal{N}, \mathcal{E})$, where $\mathcal{N}$ is a finite nonempty set of nodes and $\mathcal{E} \in \mathcal{N}^{2}$ is a set of ordered pairs of nodes, called edges. As a comparison, the pairs of nodes in an undirected graph are unordered. If there is a directed edge from node $v_{i}$ to node $v_{j}$, then $v_{i}$ is defined as the parent node and $v_{j}$ is defined as the child node. A directed path is a sequence of ordered edges of the form $\left(v_{i_{1}}, v_{i_{2}}\right),\left(v_{i_{2}}, v_{i_{3}}\right), \cdots$, where $v_{i_{j}} \in \mathcal{N}$, in a digraph. An undirected path in an undirected graph is defined accordingly. A digraph is called strongly connected if there is a directed path from every node to every other node. An undirected graph is called connected if there is a path between any distinct pair of nodes. In a digraph, a cycle is a path that starts and ends at the same node. A directed tree is a directed graph, where every node has exactly one parent except for one node, called root, which has no parent, and the root has a directed path to every other node. Note that in a directed tree, each edge has a natural orientation away from the root, and no cycle exists. A (directed) spanning tree of a digraph is a directed tree formed by graph edges that connect all the nodes of the graph. We say that a graph has (or contains) a (directed) spanning tree if there exists a (directed) spanning tree being a subset of the graph. Note that the condition that a digraph has a (directed) spanning tree is equivalent to the case that there exists a node having a directed path to all the other nodes. In the case of undirected graphs, having an undirected spanning tree is equivalent to being connected. However, in the case of digraphs, having a directed spanning tree is a weaker condition than being strongly connected.

Let $\mathbf{1}$ and $\mathbf{0}$ denote the $n \times 1$ column vector of all ones and all zeros respectively. Let $I_{n}$ denote the $n \times n$ identity matrix and $0_{m \times n}$ denote the $m \times n$ matrix with all zero entries. Let $M_{n}(\mathbb{R})$ represent the set of all $n \times n$ real matrices. Given a matrix $A=\left[a_{i j}\right] \in M_{n}(\mathbb{R})$, the digraph of $A$, denoted by $\Gamma(A)$, is the digraph on $n$ nodes $v_{i}, i \in\{1,2, \cdots, n\}$, such that there is a directed edge in $\Gamma(A)$ from $v_{j}$ to $v_{i}$ if and only if $a_{i j} \neq 0 .^{9}$

The adjacency matrix $A=\left[a_{i j}\right]$ of a weighted digraph is defined as $a_{i i}=0$ and $a_{i j}>0$ if $(j, i) \in \mathcal{E}$ where $i \neq j$. The Laplacian matrix of the weighted digraph is defined as $L=\left[\ell_{i j}\right]$, where $\ell_{i i}=\sum_{j \neq i} a_{i j}$ and $\ell_{i j}=-a_{i j}$ where $i \neq j$. For an undirected graph, the Laplacian matrix is symmetric positive semi-definite.

In the case of an undirected interaction graph, the graph Laplacian has a simple zero eigenvalue if and only if the graph is connected. ${ }^{10}$ In the case of a directed interaction graph, the digraph Laplacian has a simple zero eigenvalue and all the other eigenvalues have positive real parts if and only if the digraph has a (directed) spanning tree. ${ }^{11}$ In both cases, $\mathbf{1}$ is the eigenvector of the graph (digraph) Laplacian associated with eigenvalue zero.

\section{B. Consensus Algorithm}

Convergence to a common value is called information consensus or agreement in the literature. Let $x_{i}$ be the information state associated with the $i^{\text {th }}$ vehicle. The information state represents information that needs to be coordinated between vehicles. The information state may be vehicle position, velocity, oscillation phase, decision variable, and so on. The set of vehicles is said to achieve consensus asymptotically if for any $x_{i}(0)$, $\left\|x_{i}(t)-x_{j}(t)\right\| \rightarrow 0$ as $t \rightarrow \infty$. 
Assume that the dynamics of each vehicle are given by

$$
\dot{x}_{i}=f_{i}\left(t, x_{i}, u_{i}\right)
$$

and the control input to the $i^{\text {th }}$ vehicle is given by

$$
u_{i}=k_{i}\left(t, x_{i},\left\{j \in \mathcal{N}_{i}(t) \mid x_{j}\right\}\right),
$$

where $\mathcal{N}_{i}(t)$ represents the set of vehicles whose information is available to vehicle $i$ at time $t$. Note that the information exchange topology between vehicles may be dynamically changing in the sense that some vehicles may not have any information exchange with other vehicles during some time intervals. The goal is to design $u_{i}$ such that $\left\|x_{i}-x_{j}\right\| \rightarrow 0, \forall i \neq j$, asymptotically for any $x_{i}(0)$.

\section{Leaderless Formation Control Strategy}

Suppose that the dynamics of each vehicle are

$$
\dot{r}_{i}=v_{i}, \quad \dot{v}_{i}=u_{i},
$$

where $r_{i} \in \mathbb{R}^{m}$ and $v_{i} \in \mathbb{R}^{m}$ represent the position and velocity of vehicle $i$, and $u_{i} \in \mathbb{R}^{m}$ is the control input.

Let $v^{r}(t) \in \mathbb{R}^{m}$ be the reference velocity for the team. We propose a consensus algorithm as follows:

$$
u_{i}=\dot{v}^{r}-\alpha\left(v_{i}-v^{r}\right)-\sum_{j \in \mathcal{N}_{i}} k_{i j}\left[\left(r_{i}-r_{j}\right)+\gamma\left(v_{i}-v_{j}\right)\right],
$$

where $k_{i j}>0, \alpha>0, \gamma>0$, and $\mathcal{N}_{i}$ denotes the set of vehicles whose positions and velocities are available to vehicle $i$. Note that algorithm (2) is distributed in nature in the sense that only local neighbor-to-neighbor information exchange is required. Also note that no explicit leader is specified in this algorithm.

With the algorithm (2), Eq. (1) can be rewritten as

$$
\ddot{\tilde{r}}_{i}=-\alpha \dot{\tilde{r}}_{i}-\sum_{j \in \mathcal{N}_{i}} k_{i j}\left[\left(\tilde{r}_{i}-\tilde{r}_{j}\right)+\gamma\left(\tilde{v}_{i}-\tilde{v}_{j}\right)\right]
$$

where $\tilde{r}_{i}=r_{i}-\int_{0}^{t} v^{r} d t$ and $\tilde{v}_{i}=v_{i}-v^{r}$. It is shown in Ref. 8 that $\tilde{r}_{i} \rightarrow \tilde{r}_{j}$ and $\tilde{v}_{i} \rightarrow \tilde{v}_{j} \rightarrow 0$ if the information exchange topology has a (directed) spanning tree and $\gamma$ is sufficiently large, which implies $r_{i} \rightarrow r_{j}$ and $v_{i} \rightarrow v_{j} \rightarrow v^{r}(t)$.

A variant of consensus algorithm (2) can be used to derive local control laws for each vehicle such that they agree on a common (time-varying) formation center. Let $r_{j}$ be the $j^{\text {th }}$ vehicle's position. Let $r_{0 j}$ be the $j^{\text {th }}$ vehicle's understanding of the formation center. Also let $r_{j F}$ be the desired deviation of the $j^{\text {th }}$ vehicle from its understanding of the formation center. Note that $r_{j}=r_{0 j}+r_{j F}$. Fig. 1 shows a scenario where multiple vehicles reach consensus on a (possibly time-varying) formation center. If $r_{0 j}$ reaches a common value, denoted as $r_{0}$, then the desired formation shape is preserved since $r_{j} \rightarrow r_{0}+r_{j F}$. In this case, the control input is designed as

$$
\begin{aligned}
u_{i} & =\ddot{r}_{i F}+\dot{v}_{F}^{d}-\alpha\left(v_{i}-\dot{r}_{i F}-v_{F}^{d}\right) \\
& -\sum_{j \in \mathcal{N}_{i}} k_{i j}\left\{\left[\left(r_{i}-r_{i F}\right)-\left(r_{j}-r_{j F}\right)\right]+\gamma\left[\left(v_{i}-\dot{r}_{i F}\right)-\left(v_{j}-\dot{r}_{j F}\right)\right]\right\},
\end{aligned}
$$

where $v_{F}^{d} \in \mathbb{R}^{m}$ specifies the nominal formation velocity.

Note that $r_{i}-r_{i F}$ and $v_{i}-\dot{r}_{i F}$ satisfy consensus algorithm (2) with $r_{i}-r_{i F}, v_{i}-\dot{r}_{i F}$, and $v_{F}^{d}$ playing the role of $r_{i}, v_{i}$, and $v^{r}$ respectively. Then we know that $r_{i}-r_{i F} \rightarrow r_{j}-r_{j F}$ and $v_{i}-\dot{r}_{i F} \rightarrow v_{j}-\dot{r}_{j F} \rightarrow v_{F}^{d}$ if the information exchange topology has a (directed) spanning tree and $\gamma$ is sufficiently large. That is, $r_{0 i} \rightarrow r_{0 j}$ and $\dot{r}_{0 i} \rightarrow \dot{r}_{0 j} \rightarrow v_{F}^{d}$. 


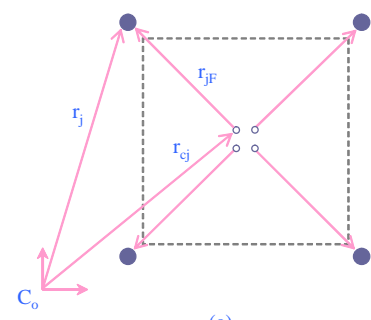

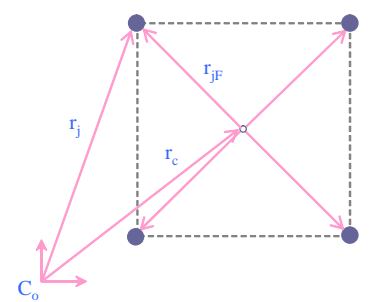

(b)

Figure 1. Consensus reached on a (possibly time-varying) formation center.

\section{Application to Formation Flying of Multiple MAVs}

In this section, we apply consensus strategy (3) to coordinate the flight of multiple rotary-wing MAVs to form a sensor web with a time-varying desired geometric configuration.

Let $\left(x_{i}, y_{i}, h_{i}\right), \psi_{i}, v_{i}, r_{i}$, and $v_{h i}$ denote the three-dimensional inertial position, heading angle, forward velocity, heading rate, and vertical velocity of the $i^{\text {th }}$ rotary-wing MAV respectively. With the MAV equipped with efficient low-level controllers, the simplified equations of motion are given by

$$
\begin{aligned}
\dot{x}_{i} & =v_{i} \cos \left(\psi_{i}\right) \\
\dot{y}_{i} & =v_{i} \sin \left(\psi_{i}\right) \\
\dot{\psi}_{i} & =r_{i} \\
\dot{v}_{i} & =\frac{1}{\alpha_{v i}}\left(v_{i}^{c}-v_{i}\right) \\
\dot{r}_{i} & =\frac{1}{\alpha_{r i}}\left(r_{i}^{c}-r_{i}\right) \\
\dot{h}_{i} & =v_{h i}, \\
\dot{v}_{h i} & =\frac{1}{\alpha_{v_{h i}}}\left(v_{h i}^{c}-v_{h i}\right)
\end{aligned}
$$

where $v_{i}^{c}, r_{i}^{c}$, and $v_{h i}^{c}$ are the commanded forward velocity, heading rate, and vertical velocity to the low-level controllers, and $\alpha_{*}$ are positive constants. ${ }^{12}$ Assuming that effective altitude-hold controllers guarantee that the MAVs fly at the same constant altitude, we will focus on the design of velocity and heading rate control commands in the following.

To avoid the nonholonomic constraint introduced by Eq. (4), we define

$$
\left[\begin{array}{l}
x_{f i} \\
y_{f i}
\end{array}\right]=\left[\begin{array}{l}
x_{i} \\
y_{i}
\end{array}\right]+\left[\begin{array}{l}
d_{i} \cos \left(\psi_{i}\right) \\
d_{i} \sin \left(\psi_{i}\right)
\end{array}\right]
$$

Note that if $\left(x_{i}, y_{i}\right)$ represents MAV $i$ 's lateral CG position in inertial coordinates, $\left(x_{f i}, y_{f i}\right)$ represents the inertial position of a point $f_{i}$ located a distance $d_{i}$ along the $x$ body axis of the $i^{\text {th }}$ MAV, presuming zero pitch angle. In the following, we will focus on the coordination of $\left(x_{f i}, y_{f i}\right)$ instead of $\left(x_{i}, y_{i}\right)$ to simplify design of the coordination algorithms.

Motivated by Ref. 13, if we let

$$
\left[\begin{array}{c}
v_{i}^{c} \\
r_{i}^{c}
\end{array}\right]=\left[\begin{array}{c}
v_{i} \\
r_{i}
\end{array}\right]+\left[\begin{array}{cc}
\alpha_{v i} & 0 \\
0 & \alpha_{r i}
\end{array}\right]\left[\begin{array}{cc}
\cos \left(\psi_{i}\right) & -d_{i} \sin \left(\psi_{i}\right) \\
\sin \left(\psi_{i}\right) & d_{i} \cos \left(\psi_{i}\right)
\end{array}\right]^{-1}\left[\begin{array}{c}
\mu_{x i}+v_{i} r_{i} \sin \left(\psi_{i}\right)+d_{i} r_{i}^{2} \cos \left(\psi_{i}\right) \\
\mu_{y i}-v_{i} r_{i} \cos \left(\psi_{i}\right)+d_{i} r_{i}^{2} \sin \left(\psi_{i}\right)
\end{array}\right],
$$

we obtain the following equations of motion:

$$
\begin{aligned}
& \dot{r}_{f i}=v_{f i} \\
& \dot{v}_{f i}=\mu_{f i},
\end{aligned}
$$


where $r_{f i}=\left[x_{f i}, y_{f i}\right]^{T}$ and $\mu_{f i}=\left[\mu_{x i}, \mu_{y i}\right]^{T}$. Note that the transformation between $\left(\mu_{x i}, \mu_{y i}\right)$ and $\left(v_{i}^{c}, r_{i}^{c}\right)$ are invertible.

We apply control law (3) to design $\mu_{f i}$ such that a team of four MAVs fly with a pre-defined formation velocity given by $v_{F}^{d}(t)$ and the team preserves a time-varying square geometric configuration during the flight.

The parameter values used in the simulation are given by Table 1, where

$$
\begin{gathered}
\lambda(t)=\left\{\begin{array}{ll}
\frac{t}{100}+\frac{1}{2}, & t<50 \mathrm{sec} \\
1, & t \geq 50 \mathrm{sec}
\end{array},\right. \\
\phi(t)= \begin{cases}0, & t<50 \mathrm{sec} \\
\frac{t-50}{15}, & 50 \leq t<50+\frac{15 \pi}{2} \mathrm{sec}, \\
\frac{\pi}{2}, & t \geq 50+\frac{15 \pi}{2} \mathrm{sec}\end{cases}
\end{gathered}
$$

and

$$
R(\phi(t))=\left[\begin{array}{cc}
\cos (\phi(t)) & \sin (\phi(t)) \\
-\sin (\phi(t)) & \cos (\phi(t))
\end{array}\right] .
$$

Note that the size of the desired square geometric configuration between the four MAVs will be expanded at $t \in[0,50)$ seconds as shown by the definitions of $\lambda(t)$ and $r_{i F}(t), i=1, \cdots, 4$.

Table 1. Parameter values used in simulation.

\begin{tabular}{|c|c|}
\hline Parameter & Value \\
\hline \hline$\alpha_{v i}$ & 1 \\
\hline$\alpha_{r i}$ & 1 \\
\hline$k_{i j}$ & 1 \\
\hline$v_{i}^{c}$ & $\in[-3,3] \mathrm{m} / \mathrm{s}$ \\
\hline$r_{i}^{c}$ & $\in[-1,1] \mathrm{rad} / \mathrm{s}$ \\
\hline$v_{F}^{d}$ & $2 *[\sin (\phi), \cos (\phi)]^{T}$ \\
\hline$r_{1 F}$ & $\lambda(t) R(\phi(t))[10,10]^{T}$ \\
\hline$r_{2 F}$ & $\lambda(t) R(\phi(t))[-10,10]^{T}$ \\
\hline$r_{3 F}$ & $\lambda(t) R(\phi(t))[-10,-10]^{T}$ \\
\hline$r_{4 F}$ & $\lambda(t) R(\phi(t))[-10,10]^{T}$ \\
\hline
\end{tabular}

The information exchange topologies between the four MAVs are given by Fig. 2, where a directed edge from the $i^{\text {th }}$ MAV to the $j^{\text {th }}$ MAV means that the $j^{\text {th }}$ MAV can receive information from the $i^{\text {th }}$ MAV. Taking into account measurements from sensors with limited fields of views or random communication data loss, we assume a unidirectional information flow topology. Note that subplot (a) in Fig. 2 has a (directed) spanning tree while subplot (b) does not have a (directed) spanning tree.

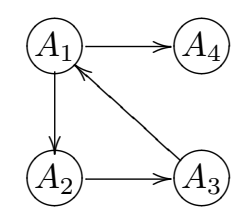

(a)

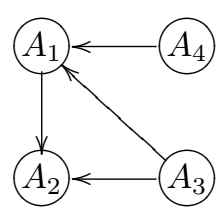

(b)

Figure 2. Information exchange topologies between the four MAVs.

We will consider three cases. Table 2 gives control parameters for each case. 
Table 2. Control parameters for different cases.

\begin{tabular}{lll}
\hline Case 1: & Interaction graph: Fig. 2 (a) & $\alpha=1, \gamma=1$ \\
Case 2: & Interaction graph: Fig. 2 (b) & $\alpha=1, \gamma=1$ \\
Case 3: & Interaction graph: Fig. 2 (a) & $\alpha=0.5, \gamma=0.05$ \\
\hline
\end{tabular}

Figs. 3, 4, and 5 show the trajectories of the four MAVs in Cases 1, 2, and 3 respectively, where squares represent the actual starting positions of each MAV respectively $(t=0 \mathrm{sec})$, circles represent the actual ending positions of each MAV $(t=100 \mathrm{sec})$, and triangles represent the actual positions of each MAV at $t=\{25 ; 50 ; 75\}$ sec. Note that the team preserves the desired time-varying square formation and flies with a nominal formation velocity given by $v_{F}^{d}$ in Case 1 . However, the desired time-varying square formation is not preserved in either Case 2 or Case 3 due to the lack of a (directed) spanning tree in Fig. 2 (b) in Case 2 and small $\gamma$ and $\alpha$ in Case 3 .

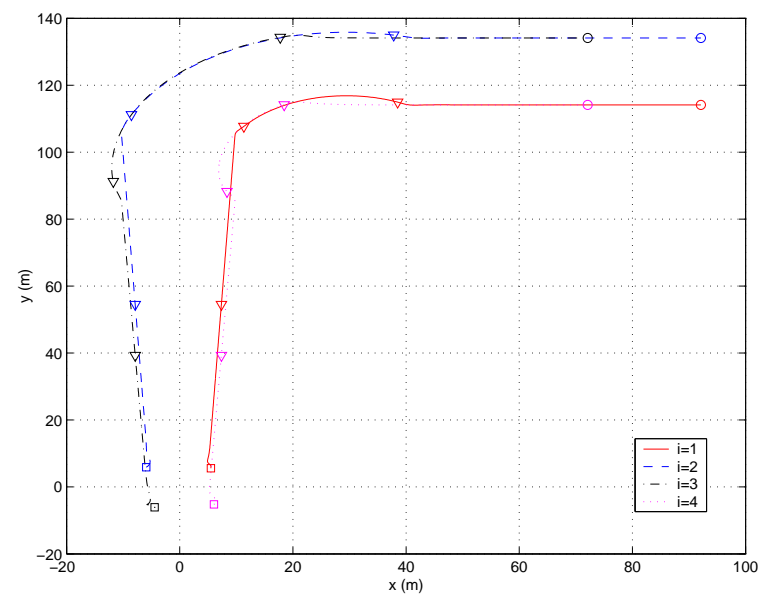

Figure 3. Trajectories of the four MAVs in Case 1.

Figs. 6, 7, and 8 show the trajectories of each MAV's understanding of the formation center, denoted by $r_{0 j}=r_{f j}-r_{j F}, j=1, \cdots, 4$, in Cases 1,2 , and 3 respectively. Note that each MAV reaches consensus on the (time-varying) formation center (i.e. $r_{0 i} \rightarrow r_{0 j}, \forall i \neq j$ ) in Case 1 . However, consensus on the formation center is not reached in either Case 2 or Case 3 due to the lack of a (directed) spanning tree in Fig. 2 (b) and small $\gamma$ and $\alpha$ respectively.

Fig. 9, 10, and 11 show the commanded forward velocities and heading rates of each MAV in Cases 1, 2 , and 3 respectively. Note that the control commands satisfy the saturation constraints defined in Table 1. The discontinuities at $t=50$ seconds are due to the fact that $\lambda(t)$ and $\phi(t)$ are not differentiable at $t=50$ seconds.

\section{Conclusion}

We have proposed a consensus-based leaderless formation control strategy for multiple autonomous vehicles. With the proposed strategy, no explicit leaders exist in the team and only local neighbor-to-neighbor information exchange is required. The proposed strategy has been applied in simulation to maintain a time-varying formation for multiple MAVs.

\section{References}

${ }^{1}$ Wang, P. K. C., "Navigation Strategies for Multiple Autonomous Mobile Robots Moving in Formation," Journal of Robotic Systems, Vol. 8, No. 2, 1991, pp. 177-195.

6 of 10 


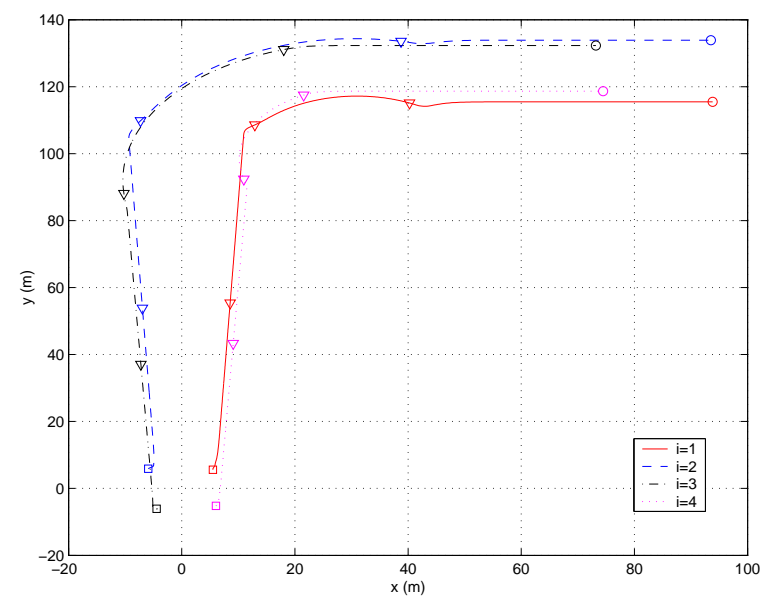

Figure 4. Trajectories of the four MAVs in Case 2.

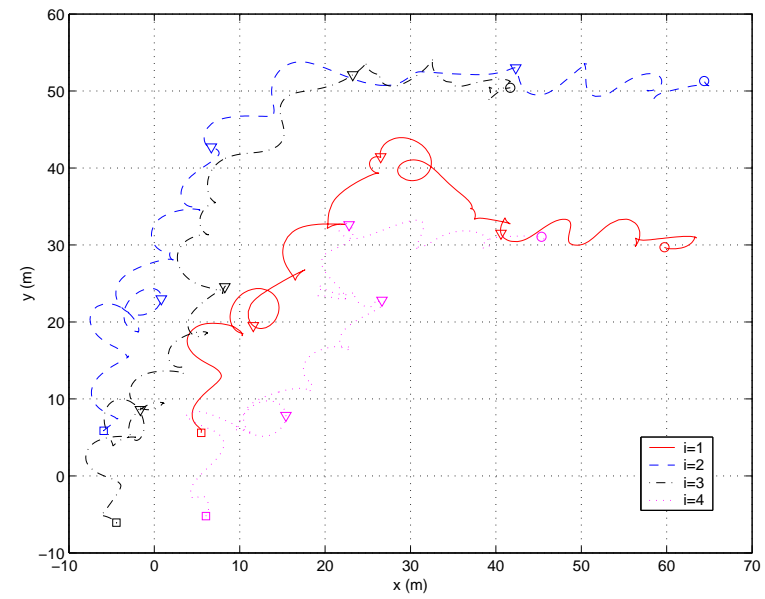

Figure 5. Trajectories of the four MAVs in Case 3.

${ }^{2}$ Wang, P. K. C. and Hadaegh, F. Y., "Coordination and Control of Multiple Microspacecraft Moving in Formation," The Journal of the Astronautical Sciences, Vol. 44, No. 3, 1996, pp. 315-355.

${ }^{3}$ Jadbabaie, A., Lin, J., and Morse, A. S., "Coordination of Groups of Mobile Autonomous Agents Using Nearest Neighbor Rules," IEEE Transactions on Automatic Control, Vol. 48, No. 6, June 2003, pp. 988-1001.

${ }^{4}$ Olfati-Saber, R. and Murray, R. M., "Consensus problems in networks of agents with switching topology and time-delays," IEEE Transactions on Automatic Control, Vol. 49, No. 9, September 2004, pp. 1520-1533.

${ }^{5}$ Ren, W. and Beard, R. W., "Consensus Seeking in Multiagent Systems Under Dynamically Changing Interaction Topologies," IEEE Transactions on Automatic Control, Vol. 50, No. 5, May 2005, pp. 655-661.

${ }^{6}$ Tanner, H. G., Jadbabaie, A., and Pappas, G. J., "Stable Flocking of Mobile Agents, Part I: Fixed Topology," Proceedings of the IEEE Conference on Decision and Control, Maui, Hawaii, December 2003, pp. 2010-2015.

${ }^{7}$ Olfati-Saber, R. and Murray, R. M., "Flocking with obstacle avoidance: Cooperation with limited communication in mobile networks," Proceedings of the IEEE Conference on Decision and Control, Maui, Hawaii, December 2003, pp. 20222028.

${ }^{8}$ Ren, W. and Atkins, E., "Second-order Consensus Protocols in Multiple Vehicle Systems with Local Interactions," Proceedings of the AIAA Guidance, Navigation, and Control Conference, San Francisco, CA, August 2005, paper no. AIAA2005-6238.

${ }^{9}$ Horn, R. A. and Johnson, C. R., Matrix Analysis, Cambridge University Press, 1985.

${ }^{10}$ Chung, F. R. K., "Spectral Graph Theory," Regional Conference Series in Mathematics, American Mathematical Society, 1997.

${ }^{11}$ Ren, W., Beard, R. W., and McLain, T. W., "Coordination Variables and Consensus Building in Multiple Vehicle Systems," Cooperative Control: A Post-Workshop Volume 2003 Block Island Workshop on Cooperative Control, edited by 


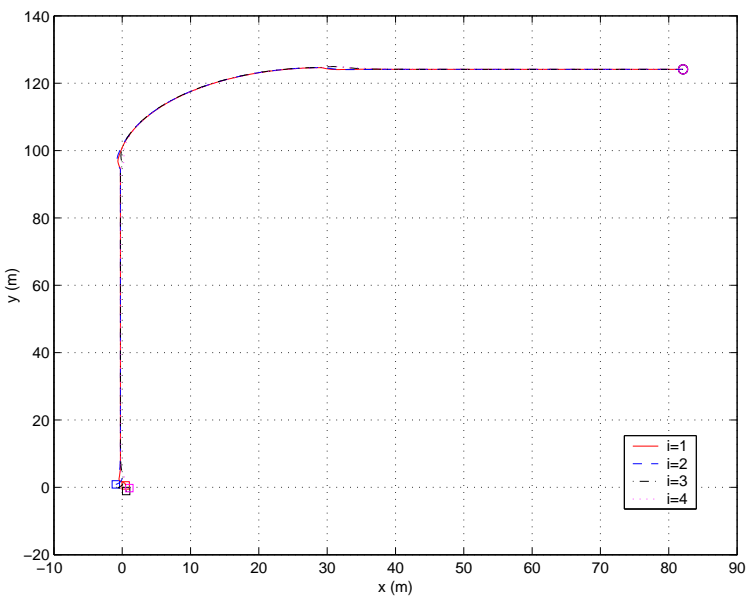

Figure 6. Trajectories of each MAV's understanding of the formation center in Case 1.

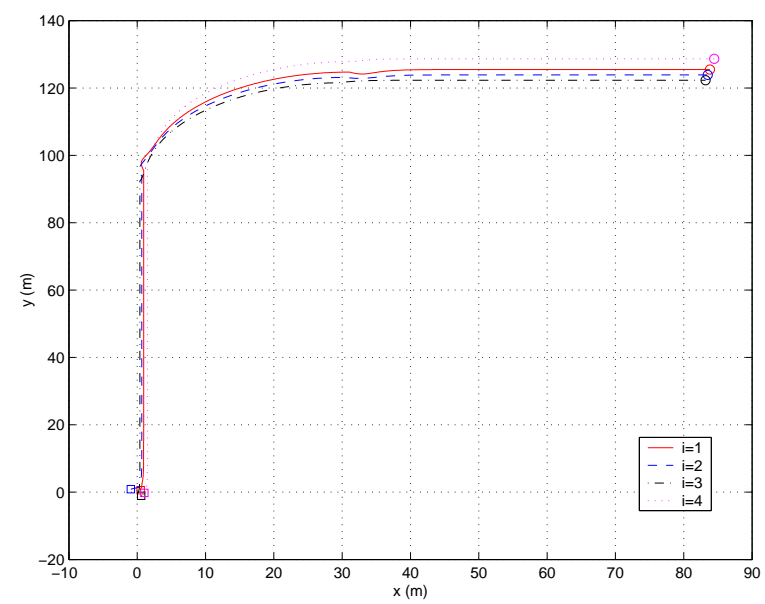

Figure 7. Trajectories of each MAV's understanding of the formation center in Case 2.

V. Kumar, N. E. Leonard, and A. S. Morse, Vol. 309, Springer-Verlag Series: Lecture Notes in Control and Information Sciences, 2004, pp. 171-188.

${ }^{12}$ Gavrilets, V., Mettler, B., and Feron, E., "Human-Inspired Control Logic for Automated Maneuvering of Miniature Helicopter," AIAA Journal of Guidance, Control, and Dynamics, Vol. 27, No. 5, September-October 2004, pp. 752-759.

${ }^{13}$ Lawton, J. R., Beard, R. W., and Young, B., "A Decentralized Approach To Formation Maneuvers," IEEE Transactions on Robotics and Automation, Vol. 19, No. 6, December 2003, pp. 933-941. 


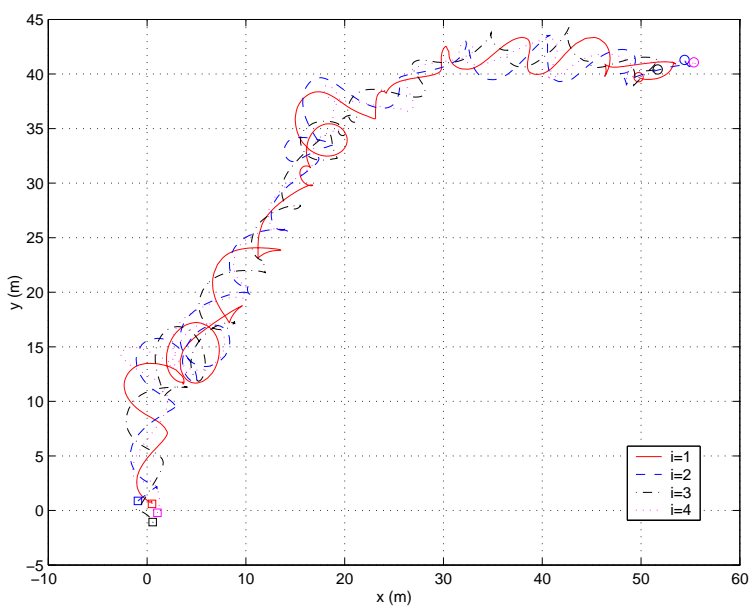

Figure 8. Trajectories of each MAV's understanding of the formation center in Case 3.
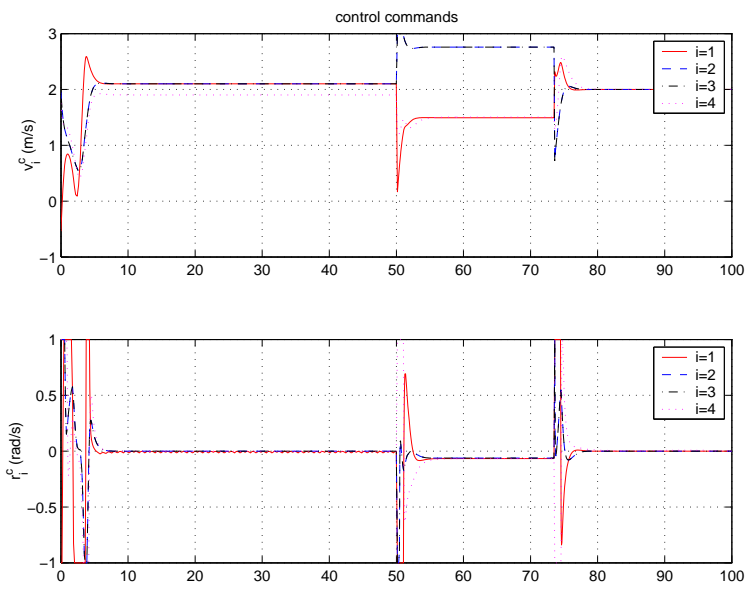

Figure 9. Control commands of each MAV in Case 1.
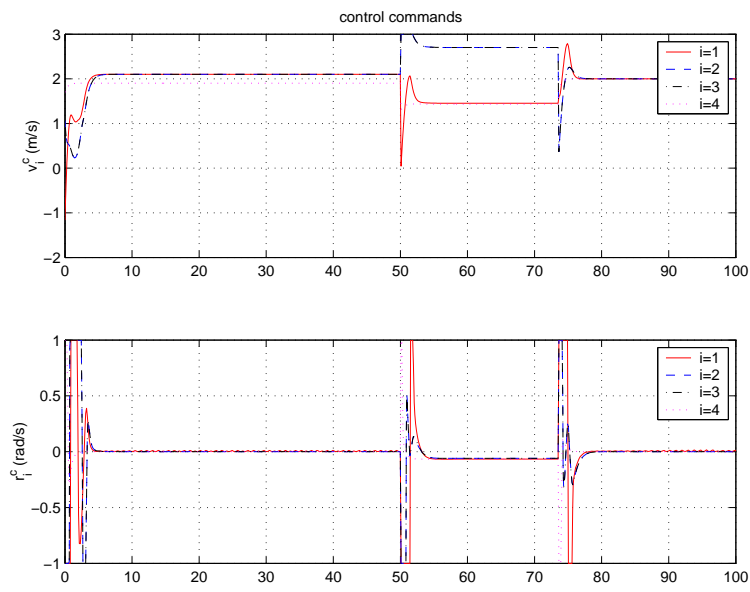

Figure 10. Control commands of each MAV in Case 2.

9 of 10

American Institute of Aeronautics and Astronautics 

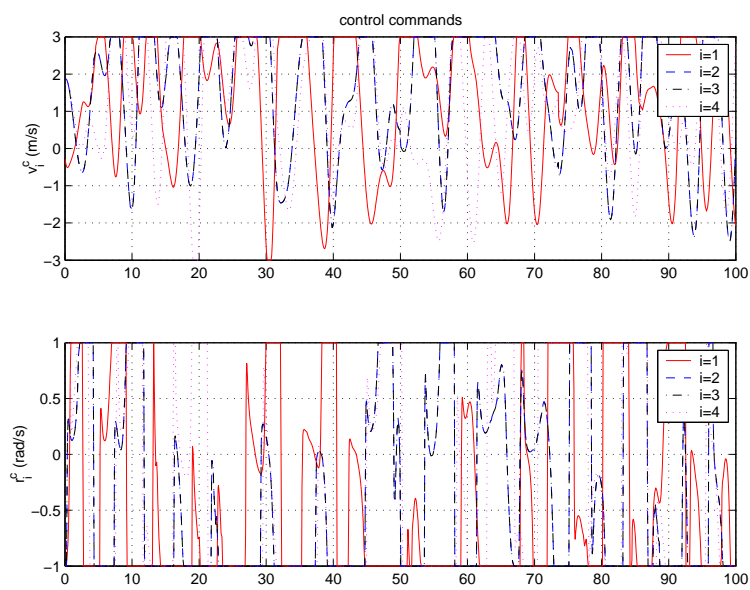

Figure 11. Control commands of each MAV in Case 3.

10 of 10 